decreased progression-free survival (PFS) and overall survival (OS), likely mediated by lower gut microbial diversity and skewed taxonomic abundance. The relationship of late antibiotic exposure to ICI efficacy has not been explored.

Methods Data from a single-arm, Phase 1/2 study (Study 1108) were retrospectively analyzed in 945 patients with metastatic disease in 18 tumor types (largest subsets: nonsmall-cell lung cancer [NSCLC], 31.5\% and urothelial cancer [UC], 20.8\%) receiving durvalumab $10 \mathrm{mg} / \mathrm{kg}$ Q2W between $8 / 2012-6 / 2017$. Early antibiotic exposure was defined as 30 days prior to until 30 days after durvalumab initiation. Late exposure was any time $>30$ days after durvalumab initiation. Demographics, infection type, and antibiotic data (route, duration, class) were collected. Median PFS and OS were compared between no antibiotics $(\mathrm{n}=525)$, early antibiotics $(n=239)$, and late antibiotics $(n=181)$ by Kaplan-Meier methods and log-rank tests. The Cox proportional hazards model was used for multivariable adjustments. In a translational in vivo analysis, Balb/c mice implanted with CT26 tumors were prospectively treated with oral levofloxacin $1 \mathrm{mg} / \mathrm{mL}$ for 7 days either 1 week prior to, concurrently with, or 1 week after initiating anti-PD-L1 or control IgG therapy $(n=40$ per group). The primary endpoint was tumor growth kinetics.

Results ß-lactams (51.3\%) and fluoroquinolones (39.3\%) were most commonly prescribed overall. Early antibiotic exposure was associated with reduced mOS compared to no exposure (7.2 vs 9.8 months, $p=0.049$ ). Unexpectedly, patients with late antibiotic exposure had markedly improved mOS versus those with no exposure (19.8 vs 9.8 months, $\mathrm{p}<0.001)$, which persisted after adjusting for baseline tumor volume, demographics, treatment-induced immune-related adverse events, and neutrophil to lymphocyte ratio (table 1). In NSCLC and UC cohorts, these results were preserved. To account for time-on-treatment bias, an independent model using antibiotic start time as a time-dependent covariate also showed improved benefit with late antibiotics (HR 0.91, 95\% CI 0.87-0.95, $\mathrm{p}<0.001)$. Compared to control $\mathrm{IgG}$, mice receiving prior or concurrent levofloxacin with anti-PD-L1 had similar survival, while mice receiving late levofloxacin with anti-PD-L1 had improved survival $(\mathrm{p}=0.004)$.

Abstract 674 Table 1 mPFS and mOS by antibiotic exposure

\begin{tabular}{|l|c|c|}
\hline Groups ( $\mathrm{n})$ & mPFS (95\% Cl), months & mOS (95\% Cl), months \\
\hline All patients (945) & $1.6(1.5-1.8)$ & $10.8(9.3-12.4)$ \\
\hline No antibiotics (525) & $1.4(1.4-1.6)$ & $9.8(7.8-11.4)$ \\
\hline Early antibiotics* (239) & $1.5(1.4-2.2)$ & $7.2(5.4-9.4)$ \\
\hline Late antibiotics** (181) & $4.4(2.8-5.6)$ & $19.8(15.5-23.0)$ \\
\hline
\end{tabular}
* Early antibiotics defined as 30 days prior to until 30 days after durvalumab initiation
** Late exposure was any time greater than 30 days after durvalumab initiation

Conclusions This large retrospective analysis is consistent with previous studies showing associations of early antibiotics with shorter survival during ICI therapy. For the first time, we report that late antibiotics do not negatively impact survival and may actually benefit survival. Future studies will evaluate immune phenotyping and microbiome characterization to clarify mechanistic underpinnings. These findings will require confirmation in prospective clinical studies.

Trial Registration clinicaltrials.gov NCT01693562

Ethics Approval This study was conducted according to the Declaration of Helsinki and approved by the independent ethics committee/institutional review board at each participating center.
Consent Informed consent was obtained from all patients.

http://dx.doi.org/10.1136/jitc-2020-SITC2020.0674

\section{OXYGEN CONCENTRATION ALTERS NATURAL KILLER CELL PHENOTYPE AND FUNCTION IN THE SOLID TUMOR MICROENVIRONMENT}

'Upasana Sunil Arvindam*, ${ }^{1}$ Phillipa Kennedy, ${ }^{1}$ Brianna Ettestad, ${ }^{1}$ Peter Hinderlie, ${ }^{1}$ Gwen Phung, ${ }^{2}$ James Lim, ${ }^{1}$ Jeff Miller, ${ }^{1}$ Martin Felices. ${ }^{1}$ University of Minnesota, Minneapolis, MN, USA; ${ }^{2}$ XCell Biosciences, San Francisco, CA, USA

Background Natural Killer (NK) cells can eliminate cancer cells through the release of cytotoxic granules triggered by interactions with natural ligands or through antibody-dependent cellular cytotoxicity (ADCC). ${ }^{1-3} \mathrm{NK}$ cell-based treatments have had therapeutic success for hematological malignancies but strategies to treat solid tumors have been limited due to immunosuppression within the tumor microenvironment (TME). ${ }^{4-6}$ An important and understudied aspect of NK cell immunosuppression is the low oxygen (hypoxia) environment created by proliferating tumor cells. We used the novel AVATAR incubator system to model oxygen levels of three key tissues that NK cells inhabit in vivo: the peripheral blood $(12 \%$ $\mathrm{O} 2)$, the bone marrow $(5 \% \mathrm{O} 2)$ and the TME (1\% O2).

Methods NK cells were incubated in the AVATAR incubators for 24 hours, 72 hours and 7 days. We conducted a mass cytometry (CyTOF) analysis to assess phenotype, flow cytometry-based assays to assess proliferation and an IncuCyte machine and immunofluorescent imaging to measure cytotoxicity of NK cells incubated at different oxygen conditions. We evaluated NK cell metabolism using Seahorse assays, gene expression using RNA-Seq and are in the process of evaluating epigenetic regulation using ATAC-Seq.

Results NK cells from the 1\% O2 condition express fewer activating receptors (CD16, NKG2D, Nkp30, Nkp46, DNAM1) and less perforin and granzyme than NK cells from the higher oxygen conditions (figure 1). NK cells in the 1\% O2 condition also have decreased aggregation of perforin and granzyme granules at the immune synapse. This translates to reduced natural cytotoxicity and ADCC responses against tumor targets (figure 2). We also observe a sharp decrease in proliferation in the $\mathrm{NK}$ cells at $1 \% \mathrm{O} 2$ (figure 3). This is

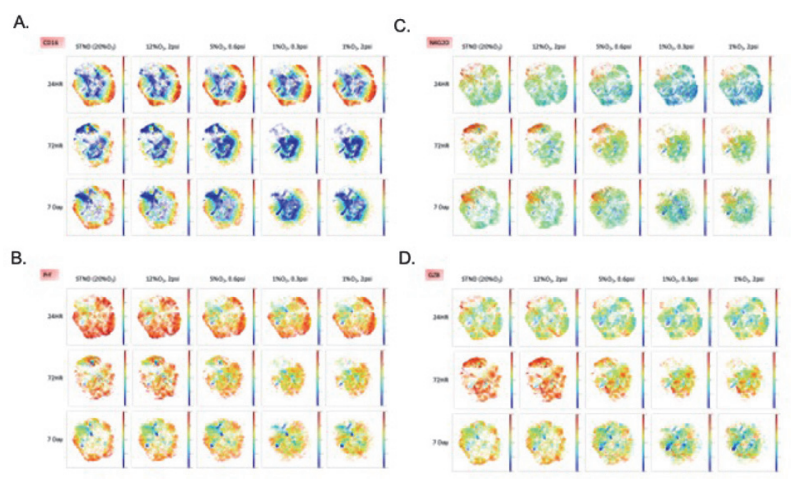

Abstract 675 Figure 1 Oxygen concentration alters NK cell phenotype

300,000 enriched NK cells were incubated for 24 hrs, 72 hrs and 7 days at noted incubator conditions with $1 \mathrm{ng} / \mathrm{ml} \mathrm{IL-15}$. At the end of the incubation, cells were barcoded and stained with a custom panel for CyTOF evaluation. Data is show here for CD16 (A), NKG2D (B), Perforin (C) and Granzyme B (D). N=3 (data concatenated). 
partly due to an increase in CISH gene expression that makes the cells less responsive to cytokine stimulation. ${ }^{7}$ The RNASeq analysis revealed that NK cell metabolism closely resembles cancer cell metabolism under hypoxic conditions, specifically an increased expression of genes related to glycolysis, amino acid synthesis and central carbon metabolism. This change in metabolism was confirmed using Seahorse assays. We also observed changes in genes related to epigenetic regulation specifically, increases in histone demethylases and decreases in DNA methyltransferases (figure 4).

Conclusions These results indicate that NK cells who enter the solid TME are fundamentally different than those in the bone marrow or the blood stream. Overall, the insights gained from these experiments can help overcome hypoxia induced

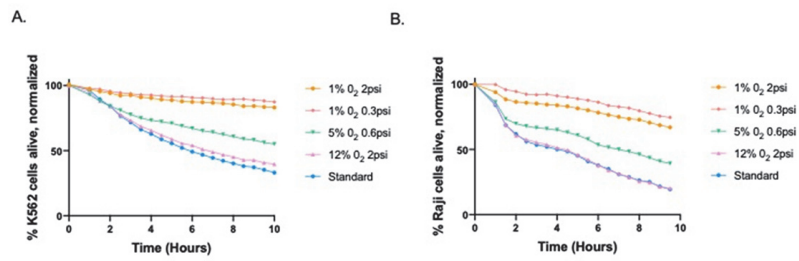

Abstract 675 Figure 2 Oxygen concentration effects NK cell cytotoxicity

300,000 enriched NK cells were plated per well in 96-well roundbottom plates with $1 \mathrm{ng} / \mathrm{ml} \mathrm{IL-15}$. Plates were inserted in noted incubator conditions for 24 hours, 72 hours or 7 days. At the end of the incubation period NK cells were counted and plated at a 2:1 E:T ratio with fluorescently labeled $\mathrm{K} 562$ targets or fluorescently labeled labeled Raji targets + Rituximab (10 ug/ml) and cells were imaged every 30 minutes. Data is shown here for K562 targets (A) and raji targets (B) at the 7 day timepoint. Representative of six separate experiments.

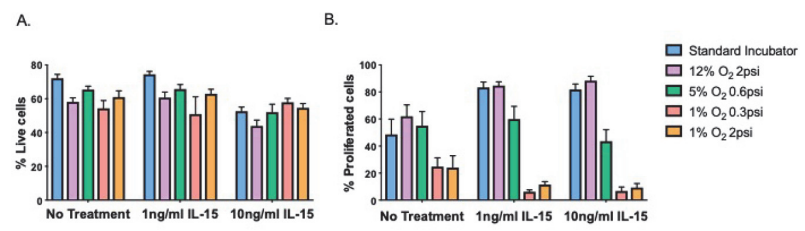

Abstract 675 Figure 3 Oxygen concentration impacts NK cell proliferation

300,000 PMBCs were CellTrace labeled and plated per well in 96-well round-bottom plates with noted treatments. The NK cells were incubated under noted incubator conditions for 7 days. At the end of 7 days, LiveDead dye was used to assess viability (A), while proliferation was assessed by evaluating CellTrace dye dilution on gated (CD56 + CD3-) NK cells (B). $(\mathrm{N}=6)$

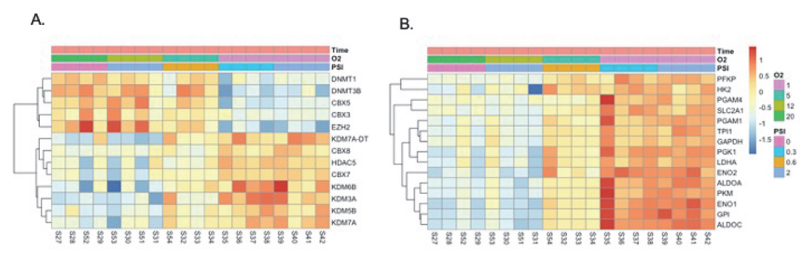

Abstract 675 Figure 4 RNA-Seq reveals changes in gene expression An RNA-Seq analysis was performed on enriched NK cells incubated in noted oxygen and pressure concentrations for 24 hours, 72 hours or 7 days. A heat map of epigenetic regulation genes (A) and glycolysis genes $(B)$ are shown for the day 7 timepoint. $(\mathrm{N}=4)$ immune suppression in the tumor microenvironment and improve NK cell-based immunotherapy for solid tumors.

Acknowledgements We thank XCell biosciences for providing us with the AVATAR incubators used for these experiments

Trial Registration N/A

Ethics Approval N/A

Consent N/A

\section{REFERENCES}

1. Vivier $E$, Tomasello $E$, Baratin $M$, Walzer $T$, Ugolini $S$. Functions of natural killer cells. Nat Immunol 2008;9(5):503-10.

2. Waldhauer I, Steinle A. NK cells and cancer immunosurveillance. Oncogene. 2008;27(45):5932-43.

3. Voskoboinik I, Smyth MJ, Trapani JA. Perforin-mediated target-cell death and immune homeostasis. Nat Rev Immunol 2006;6(12):940-52.

4. Miller JS, Soignier Y, Panoskaltsis-mortari A, Mcnearney SA, Yun GH, Fautsch SK, et al. Successful adoptive transfer and in vivo expansion of human haploidentical NK cells in patients with cancer. Blood 2005;105(8):3051-8.

5. Romee $\mathrm{R}$, Cooley $\mathrm{S}$, Berrien-Elliott MM, Westervelt $\mathrm{P}$, Verneris MR, Wagner JE, et al. First-in-human phase 1 clinical study of the IL-15 superagonist complex ALT803 to treat relapse after transplantation. Blood 2018:131(23):2515-2527.

6. Björklund AT, Carlsten M, Sohlberg E, Liu LL, Clancy T, Karimi M, et al. Complete remission with reduction of high-risk clones following haploidentical NK-Cell therapy against MDS and AML. Clin Cancer Res 2018;24(8):1834-1844.

7. Delconte RB, Kolesnik TB, Dagley LF, Rautela J, Shi W, Putz EM, et al. CIS is a potent checkpoint in NK cell-mediated tumor immunity. Nat Immunol 2016;17 (7):816-24.

http://dx.doi.org/10.1136/jitc-2020-SITC2020.0675

\section{PD-L1 IS INDUCED BY THE PERIODONTAL PATHOGEN PORPHYROMONAS GINGIVALIS AND CAN BE BLOCKED BY SMALL MOLECULE GINGIPAIN INHIBITORS, INCLUDING ATUZAGINSTAT}

Shirin Arastu-Kapur*, Mai Nguyen, Sean Broce, Joseph Vacca, Kirk Ehmsen, Florian Ermini, Ursula Haditsch, Aurora Martinez-Horta, Debasish Raha, Leonardo Rodriguez, Casey Lynch, Stephen Dominy, Leslie Holsinger. Cortexyme, Inc., South San Francisco, CA, USA

Background The periodontal pathogen Porphyromonas gingivalis ( $\mathrm{Pg})$ has been linked to esophageal and other cancers through epidemiology studies. Pg's protease virulence factors known as gingipains have been identified in esophageal cancer tissue and correlate with worse disease prognosis. Anti-PD-1 antibodies have shown some success in esophageal cancer treatment, but further understanding of the induction of PDL1 in esophageal cells is needed to identify potential treatment modalities. Pg has been shown to induce PD-L1 on the surface of infected cells, suggesting that the presence of $\mathrm{Pg}$ in esophageal cancer cells may contribute to PD-L1 expression and immune escape. One of the pathways known to induce PD-L1 is wnt pathway activation resulting in b-catenin translocation to the nucleus. Prior studies have demonstrated that Pg activates the wnt pathway by a non-canonical mechanism, leading to b-catenin nuclear localization.

Methods An immortalized non-transformed esophageal cell line, Het-1A, was used to investigate the level of PD-L1 induction by $\mathrm{Pg}$ infection using quantitative immunofluorescence. PD-L1 expression was measured using irreversible gingipain inhibitors against lysine-gingipain (Kgp) and argininegingipain (Rgp). Pg-induced PD-L1 expression pathways were investigated by Western blot and qPCR. PD-L1 induction by Pg was characterized in cancer cell lines that have an endogenous level of PD-L1 expression, including tongue squamous cell carcinoma (SCC25) and neuroblastoma (SH-SY5Y). PD-L1 induction by $\mathrm{Pg}$ was assessed in a murine derived RAW macrophage cell line that is critical for anti-PD-1 responses. 